\title{
Locus Coeruleus Neuron Growth Cones and Spinal Cord Regeneration
}

\author{
STEPHEN J. MOORMAN ${ }^{1}$ AND RICHARD 1. HUME
}

\author{
Department of Biology, Natural Science Building, The University of Michigan, Ann Arbor, MI 48109
}

\begin{abstract}
MOORMAN, S. J. AND R. I. HUME. Locus coeruleus neuron growth cones and spinal cord regeneration. BRAIN RES BULL 35(5/6) 419-422, 1994. - One of the major impediments to successful recovery of function after a spinal cord injury is thought to be the reaction of the neuronal growth cone to inhibitory influences in the local environment in or around the site of the injury. The growth cones of locus coeruleus neurons studied in vitro collapsed upon contact with an extract of CNS myelin but did not collapse on contact with an extract of PNS myelin. Coincident with the collapse of the growth cone, was an increase in internal free calcium concentration that was predominantly the result of an influx of calcium through channels in the plasma membrane. Omega-conotoxin, which specifically blocks $\mathrm{N}$-type voltage-gated calcium channels, blocked both the myelin-induced calcium influx and the coincident collapse of the growth cone.
\end{abstract}

Locus coeruleus Growth cone Myelin Calcium Omega-conotoxin Calcium channels

IF there is going to be recovery of function in an animal that has suffered an injury to the spinal cord, several things have to happen: a) neurons capable of growing axons across the site of injury must survive; b) these neurons have to generate new growth cones; c) the growth cones have to navigate either around or through the site of the injury; d) the growth cones must recognize and synapse with their appropriate target cells. In the last decade the processes of neurogenesis, axonogenesis, growth cone navigation, and synaptogenesis in the developing nervous system have received extensive scrutiny. In a number of cases, detailed control mechanisms have been elucidated at the cellular and molecular level. One example is the identification of molecules thought to play a role in the specific guidance of axons to their targets (see 9). A second example is the substantial body of evidence indicating that changes in the level of intracellular free calcium can play an important role in the rate at which growth cones extend (see 17). Our experiments are motivated by the hypothesis that many of the cellular and molecular mechanisms that have been shown to be important during neuronal development will also prove to be relevant to neuronal regeneration.

In this article we concentrate on a growth cone behavior referred to as growth cone collapse. A variety of neurons studied in vitro have been demonstrated to undergo collapse (see 18). Growth cone collapse is characterized by a relatively rapid change in the morphology of the growth cone: filopodial movements cease; there is a general loss of filopodia; the fine structure of the growth cone appears to draw back into the growth cone so that the overall surface area of the growth cone decreases; and the growth cone and distal neurite often retract. Growth cone collapse can occur spontaneously, but most commonly collapse is the result of contact with specific types of cells (see 18). Cell surface molecules that are potential signals triggering collapse are currently under intensive scrutiny. Although a great deal of progress has been made in understanding the cellular and molecular mechanisms that trigger growth cone collapse in some model systems, an important caveat to all studies in this area is that there is no conclusive evidence that growth cone collapse occurs in vivo during development. It may be some time before such information is available because imaging of elongating axons in vivo is technically very difficult.

Growth cone collapse may be relevant to the lack of regeneration in the spinal cord. It is a long-standing observation that many neurons survive an acute injury to the spinal cord and can begin to reform growth cones, but that these growth cones have great difficulty traversing the damaged area (see 13). Studies over the last decade have suggested a possible mechanism for these results. Because axons grow readily through grafts of peripheral nerve, the implication was that something specific to the damaged central nervous system was inhibitory to regrowth. By systematically varying the types of cells present, the conclusion was reached that oligodendrocytes might be associated with the inhibitory process (2). This inference has been greatly strengthened by two lines of observation. First, it has been shown that an extract of CNS myelin can elicit the collapse of the growth cones of dorsal root ganglion neurons (DRG) in vitro (3) but that PNS myelin does not elicit collapse. Second, when CNS-myelin is pretreated with antibodies against two CNS myelin-specific proteins, collapse of DRG growth cones is no longer evoked (4). These results suggested the hypothesis that the site of injury in the spinal cord might present an environment that is inhibitory to regenerating growth cones. This hypothesis was supported by the observation that the same antibodies that prevent the collapse of DRG growth cones in vitro also significantly enhance spinal cord regeneration after injury in vivo (25). Therefore, an under-

\footnotetext{
' Requests for reprints should be addressed to Stephen J. Moorman at his current address: Department of Anatomy and Cell Biology, University of North Texas, Health Science Center at Forth Worth, 3500 Camp Bowie Blvd., Fort Worth, TX 76107.
} 
standing of the cellular mechanisms that underlie growth cone collapse in response to CNS myelin might lead to the ability to enhance the growth cone's ability to successfully navigate through or around the site of a spinal cord injury.

Some (1), but not all $(7,11)$ cases of growth cone collapse are associated with a change in intracellular free calcium $\left([\mathrm{Ca}]_{i}\right)$. The specific CNS myelin proteins known to induce collapse of the growth cones of dorsal root ganglion neurons have been shown to cause a release of calcium from internal stores (1). Although these experiments demonstrated that a rise in calcium is highly correlated with the collapse mediated by CNS myelin, they did not demonstrate that this rise is necessary or sufficient for the collapse to occur. Such a demonstration would be an important next step.

The existing studies of growth cone collapse also suffer a significant drawback in extrapolating their relevance to spinal cord injury. Most of the experiments have been done using a model system (neurons from the peripheral nervous system) rather than cells that would actually be affected by injury. Because the responses of different types of neurons to a common stimulus can vary dramatically (4), we felt that it was important to study neurons whose cell bodies lie in the CNS and whose axons would probably be damaged by a spinal cord injury. We choose to study growth cones of locus coeruleus neurons from the neonatal rat brain stem. One reason for this choice is that these cells can be readily identified in cell culture (16). In addition, the noradrenaline produced by locus coeruleus neurons and released in the lumbar region of the spinal cord has well-defined effects on spinal cord reflexes $(12,21)$ and locomotion (6). This means that ultimately it should be possible to monitor recovery of function due to regeneration of locus coeruleus neurons in vivo. We also felt it necessary to demonstrate a causal relationship between any $[\mathrm{Ca}]_{i}$ change and the collapse of the growth cone.

In this article we summarize our recent results, which are described in detail elsewhere (20). We then attempt to put our work into context, and discuss its potential ramifications.

\section{RESULTS}

\section{Growth Cones of Rat Locus Coeruleus Neurons Collapsed on Contact With CNS Myelin}

Video microscopic observations were made of neonatal rat locus coeruleus neurons maintained at $37^{\circ} \mathrm{C}(18)$. Once a stable growth rate had been established, myelin extract (22) was puffed onto the growth cone. After a 60 -min period the myelin was washed off, and the size and position of each growth conc was recorded. By $60 \mathrm{~min}$ after application of CNS myelin extract, $75 \%$ of the growth cones $(n=16)$ were collapsed (Fig. 1A). The average change in surface area during the hour of contact was $-73.2( \pm 5.6) \%(n=16)$. Neither heat-inactivated CNS myelin $(n=5)$ nor trypsin-trealed CNS myelin $(n=5)$ evoked growth cone collapse. In contrast to the effects of CNS myelin, none of the growth cones $(n=5)$ collapsed after 60 min of contact with an extract of myelin from the adult rat ischiatic nerve (PNS myelin extract) prepared in an identical manner to the CNS myelin extract.

\section{CNS Myelin Evoked an Increase in Intracellular Free Calcium}

Application of CNS myelin extract to the growth cones of these neurons after they had been loaded with FURA2 (9) consistently caused a large, transient, increase in $[\mathrm{Ca}]_{i}$. Within the first 6 min after application of CNS myelin, an elevation in intracellular free calcium was observed in $75 \%$ of the growth
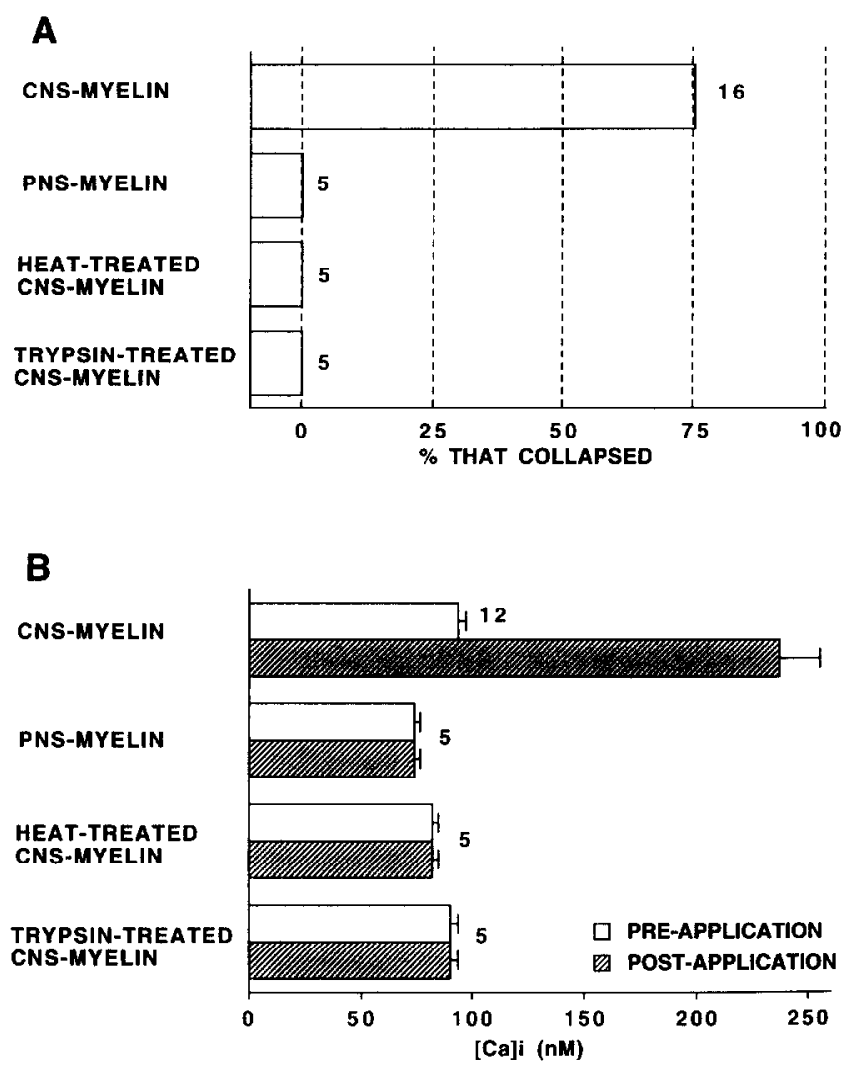

FIG. 1. (A) The percent of locus coeruleus growth cones that collapsed after application of myelin extracts. (B) The calcium concentration in locus coeruleus growth cones before and after application of myelin extracts. Number $=N$ for each group.

cones studied ( $n=12$ ). On average, the resting intracellular free calcium more than doubled from $94 \mathrm{nM}$ to $237 \mathrm{nM}$ (Fig. 1B).

Treatments that failed to evoke growth cone collapse also failed to elicit a rise in intracellular free calcium. Thus, PNS myelin, heat-inactivated myelin, and trypsin-treated myelin all had no effect on the level of intracellular free calcium (Fig. 1B).

The myelin-evoked increase in $[\mathrm{Ca}]_{i}$ was blocked when calcium was removed from the extracellular solution (by chelation with $5 \mathrm{mM}$ EGTA) and when a nonspecific blocker of several types of transmembrane calcium channels $\left(5 \mathrm{mM} \mathrm{CoCl}_{2}\right.$ ) was present in the extracellular solution. These data suggested that the increase in $[\mathrm{Ca}]_{i}$ was the result of an influx of calcium through the plasma membrane.

\section{Omega-Conotoxin Blocked the Myelin-Fvoked Increase in} $[\mathrm{Ca}]_{\mathrm{i}}$ and Prevented the Myelin-Evoked Growth Cone Collapse

To further test the role of calcium influx in causing growth cone collapse, drugs that block voltage-gated calcium channels were tested. Nifedipine, a blocker of L-type voltage-gated calcium channels, significantly reduced the magnitude of the $[\mathrm{Ca}]_{i}$ increase evoked by contact with myelin (Fig. 2A). However, even with $50 \mu \mathrm{M}$ nifedipine, a concentration that would be expected to be saturating (17), myelin extract was able to significantly increase intracellular free calcium (Fig. 2A). In media that contained $50 \mu \mathrm{M}$ nifedipine, $53 \%$ of the growth cones $(n=15)$ 

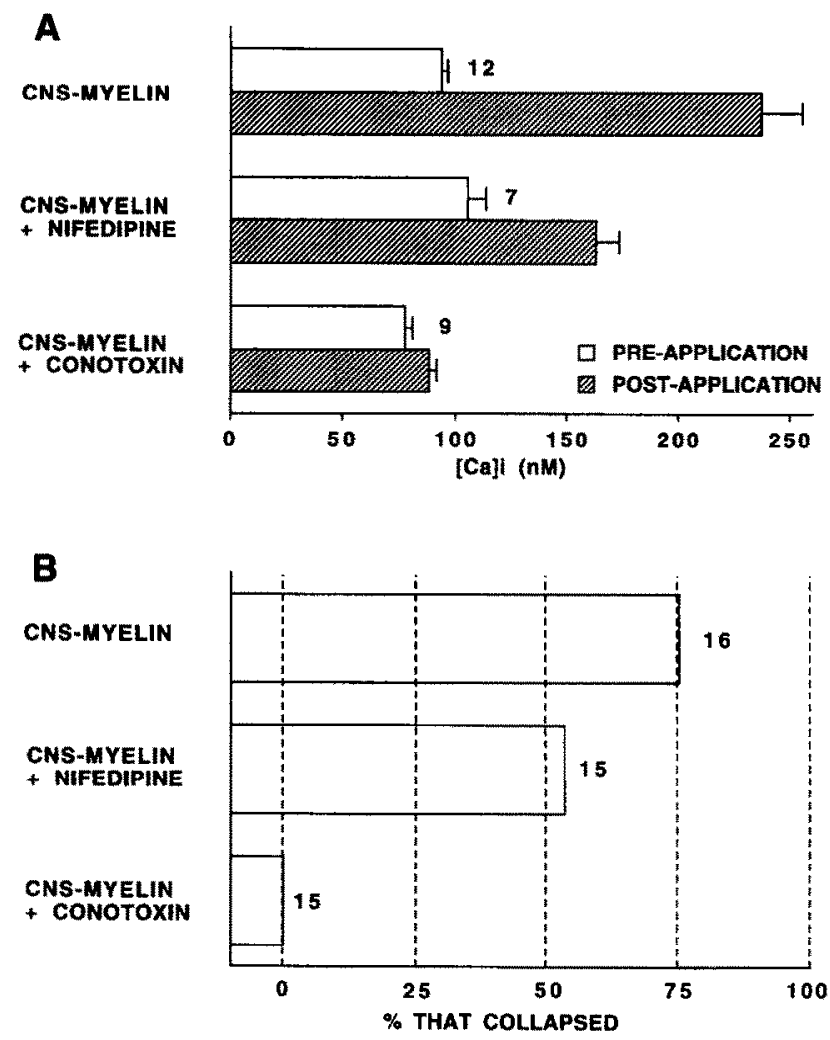

FIG. 2. (A) The calcium concentration in locus coeruleus growth cones before and after application of myelin extracts. (B) The percent of locus coeruleus growth cones that collapsed after application of myelin extracts. Number $=N$ for each group. In these experiments nifedipine was applied at $50 \mu \mathrm{M}$ and conotoxin at $5 \mathrm{nM}$.

collapsed within 60 min after contact with CNS myelin (Fig. 2B). This is not very different from the control condition where $75 \%$ collapsed after contact with CNS myelin.

In contrast, $5 \mathrm{nM}$ omega-conotoxin (23) was able to nearly completely suppress the myelin-evoked increase in $[\mathrm{Ca}]_{i}$ (Fig. $2 \mathrm{~A}$ ). Conotoxin also completely suppressed the inhibitory effect of CNS myelin on growth cone morphology and elongation. When CNS myelin was puffed onto growth cones they continued to elongate, and when the growth cones were observed at $60 \mathrm{~min}$ after the myelin was added, none of the growth cones $(n=15)$ were collapsed (Fig. 2B).

\section{DISCUSSION}

The ability of omega-conotoxin to block both the CNS myelin-induced increase in $[\mathrm{Ca}]_{i}$ and growth cone collapse is certainly suggestive of a role for calcium influx through $\mathrm{N}$-type calcium channels in growth cone collapse in locus coeruleus neurons. However, Bandtlow et al. (1) reported a purified myelin protein appears to elicit DRG growth cone collapse in vitro by inducing calcium release from internal stores, and that transmembrane flux appears to play a minor role. Furthermore, omega- conotoxin did not block the growth cone collapse induced in DRG neurons by this protein. These results suggest that the sequence of events that lead to growth cone collapse may not be the same in all cells. This point might be particularly relevant when comparing the response of neurons from the CNS to the response of neurons from the PNS to identical stimuli.

Alternatively, multiple mechanisms that can lead to growth cone collapse might exist in a single class of cells. Recently, pertussis toxin-sensitive G-proteins have been suggested to be involved in the pathway that induces collapse of growth cones from some types of neurons in response to one specific CNS myelin protein known to induce growth cone collapse (10). This potential involvement of $G$ proteins-coupled second-messenger pathways is interesting because G-proteins have also been implicated in pathways leading to a release of calcium from internal stores (26) and also in the modulation of certain ion channels in the plasma membrane $(15,24)$. It is possible that two different G-protein-linked pathways are activated by the same myelin protein, perhaps acting at different concentrations. For instance, at high protein concentrations, the G-protein-coupled release of calcium from internal stores might predominate, and at low protein concentrations the G-protein-coupled modulation of voltage-gated calcium channels might predominate. The end result in either case would be growth cone collapse. To distinguish the physiologically relevant pathway, it clearly is of importance to determine the effective concentration of active material in situ. It is also of interest that pertussis toxin treatment blocks collapse of the neuronal growth cone in response to an unidentified collapsing factor isolated from chick brain (10). This growth cone collapse is believed not to involve a coincident increase in [Ca $]_{i}(11)$. These results suggest a more general role for $\mathbf{G}$-protein - coupled secondmessenger systems in mediating growth cone collapse.

It is also possible that there are multiple myelin components that can induce cytoskeletal rearrangements that lead to collapse of the neuronal growth cone. For instance, in oligodendrocytes in vitro, a similar collapse of the leading edge (the structure analogous to the growth cone) can be induced by contact with either CNS or PNS myelin (19). In addition, antibodies against several specific components of $\mathrm{CNS}$ myelin induce changes in $[\mathrm{Ca}]_{\text {; }}$ and or cytoskeletal rearrangements in oligodendrocytes in vitro (5). However, there are only two proteins in myelin that are known to induce collapse of the neuronal growth cone in vitro $(1,3,4,27)$. Although, it should be noted that most of the work studying the affects of specific myelin proteins on the neuronal growth cone has been done on the growth cones of DRG neurons. Therefore, it remains possible that there are other components of myelin from the CNS that have similar effects on locus coeruleus neuron growth cones in vitro.

In conclusion, the mechanism by which myelin extract elicits the collapse of the growth cones of locus coeruleus neurons remains unclear. However, the observation that treatment with omega-conotoxin blocks both the $[\mathrm{Ca}]_{i}$ increase and the growth cone collapse and that treatment with pertussis toxin blocks the growth cone collapse of neurons in vitro cannot be ignored, because these suggest possible new approaches to encouraging CNS axonal regeneration in vivo.

\section{ACKNOWLEDGEMENTS}

This work was supported in part by the N.I.H., the American Paralysis Association, and a Young Investigator Fellowship (S.J.M.) from the Marine Biological Laboratory at Woods Hole.

\section{REFERENCES}

1. Bandtlow, C. E; Schmidt, M. F; Hassinger, T. D.; Schwab, M. E; Kater, S. B. Role of intracellular calcium in NI-35-evoked collapse of neuronal growth cones. Science 259:80-83; 1993.
2. Bandtlow, C. E.; Zachleder, T.; Schwab, M. E. Oligodendrocytes arrest neurite growth by contact inhibition. J. Neurosci. 10:3837$3848 ; 1990$. 
3. Caroni, P.; Schwab, M. E. Two membrane protein fractions from rat central myelin with inhibitory properties for neurite growth and fibroblast spreading. J. Cell. Biol. 106:1281-1288; 1988.

4. Caroni, P.; Schwab, M. E. Antibody against myelin-associated inhibitor of neurite growth neutralizes nonpermissive substrate properties of CNS white matter. Neuron 1:85-96; 1988.

5. Dyer, C. A. Novel oligodendrocyte transmembrane signaling systems. Investigations utilizing antibodies as ligands. Mol. Neurobiol. 7:1-22; 1993 .

6. Forssberg, H.; Grillner, S. The locomotion of the acute spinal cat injected with clonidine i.v. Brain Res. 50:184-186; 1973.

7. Garyantes, T. K.; Regehr, W. G. Electrical activity increases growth cone calcium but fails to inhibit neurite outgrowth from rat sympathetic neurons. J. Neurosci. 12:96-103; 1992.

8. Grenningloh, G.; Goodman, C. S. Pathway recognition by neuronal growth cones: Genetic analysis of neural cell adhesion molecules in Drosophila. Curr. Opin. Neurobiol. 2:42-47; 1992.

9. Grynkiewicz, G.; Poenie, M.; Tsien, R. Y. A new generation of calcium indicators with greatly improved fluorescent properties. J Biol. Chem. 260:3440-3450; 1985.

10. Igarashi, M.; Strittmatter, S. M.; Vartanian, T.; Fishman, M. C. Mediation by $\mathrm{G}$ proteins of signals that cause collapse of growth cones. Science 259:77-80; 1993.

1 1. Ivins, J. K.; Raper, J. A.; Pittman, R. N. Intracellular calcium levels do not change during contact-mediated collapse of chick DRG growth cone structure. J. Neurosci. 11:1597-1608; 1991.

12. Jankowska, E.; Jukes, M. G. M.; Lund, S.; Lundberg, A. The effect of DOPA on the spinal cord; 5 . Reciprocal organization of pathways transmitting excitatory action to alpha motoneurons of flexors and extensors. Acta Physiol. Scand. 70:369-388; 1967.

13. Kater, S. B.; Mills, L. R. Regulation of growth cone behavior by calcium. J. Neurosci. 11:891-899; 1991.

14. Keynes, R. J.; Cook, G. M. W. Repellent cues in axon guidance. Curr. Opin. Neurobiol. 2:55-59; 1992.

15. Kleuss, C.; Scherubl, H.; Hescheler, J.; Schultz, G.; Wittig, B. Selectivity in signal transduction determined by gamma subunits of heterotrimeric G proteins. Science 259:832-834; 1993.
16. Masuko, S.; Nakajima, Y.; Nakajima, S.; Yamaguchi, K. Noradrenergic neurons from the locus coeruleus in dissociated cell culture; Culture methods, morphology, and electrophysiology. J. Neurosci. $6: 3229-3241 ; 1986$.

17. McCleskey, E. W.; Fox, A. P.; Feldman, D.; Tsien, R. W. Different types of calcium channels. J. Exp. Biol. 124:177-190; 1986

18. Moorman, S. J.; Hume, R. I. Growth cones of chick sympathetic preganglionic neurons in vitro interact with other neurons in a cellspecific manner. J. Neurosci. 10:3158-3163; 1990.

19. Moorman, S. J.; Hume, R. I. Contact with myelin evokes a release of calcium from internal stores in neonatal rat oligodendrocytes invitro. GLIA 10:202-210; 1994

20. Moorman, S. J.; Hume, R. I. Omega-conotoxin prevents myelinevoked growth cone collapse in neonatal-rat locus coeruleus neurons in vitro. J. Neurosci. 13:4727-4736; 1993

21. Moorman, S. J.; Whalen, L. R. A model system to determine the effects of spccific neurotransmitters on segmental reflexes in the spinal cord of the rat. J. Neurosci. Methods 46:73-81; 1993.

22. Norton, W. T. Isolation of myelin from nerve tissue. In: Fleischer, S.; Packer, L., eds. Methods in enzymology, vol. 31. New York: Academic Press; 1976:435-444.

23. Olivera, B. M.; Cruz, L. J.; de Santos, V.; LeCheminant, G. W.; Griffin, D.; Zeikus, R.; Mclntosh, J. M.; Galyean, R.; Varga, J.; Gray, W. R. Neuronal calcium channel antagonists. Discrimination between calcium channel subtypes using omega-conotoxin from Conus magnus venom. Biochemistry 26:2086-2090; 1987.

24. Rosenthal, W.; Hescheler, J.; Hinsch, K. D.; Spicher, K.; Trautwein, W.; Schultz, G. Cyclic AMP-independent, dual regulation of voltage-dependent $\mathrm{Ca}^{2+}$ currents by LHRH and somatostatin in a pituitary cell line. EMBO J. 7:1627-1633; 1988.

25. Schnell, L.; Schwab, M. E. Axonal regeneration in the rat spinal cord produced by an antibody against myelin-associated neurite growth inhibitors. Nature 343:269-272; 1990.

26. Schuch, U.; Lohse, M.; Schachner, M. Neural cell adhesion molecules influence second messenger systems. Neuron 3:13-20; 1989.

27. Schwab, M. E. Myelin-associated inhibitors of neurite growth and regeneration in the CNS. Trends Neurosci. 13:452; 1990. 